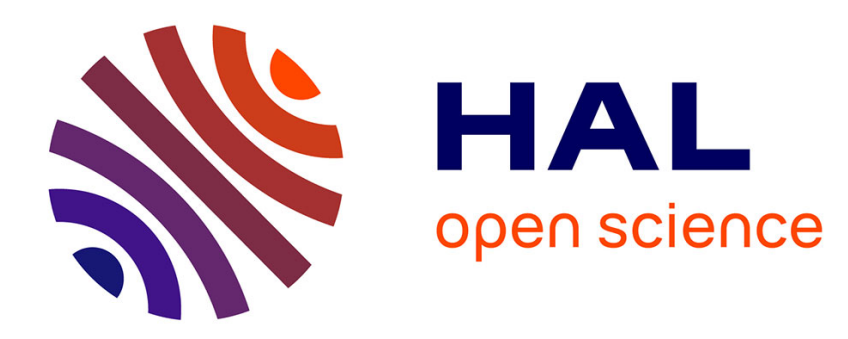

\title{
Jacoud, Gilles. Money and Banking in Jean-Baptiste Say's Economic Thought
}

\author{
Alain Béraud
}

\section{To cite this version:}

Alain Béraud. Jacoud, Gilles. Money and Banking in Jean-Baptiste Say's Economic Thought. European Journal of the History of Economic Thought, 2013, 20 (4), pp.680-683. 10.1080/09672567.2013.819966 . halshs-00865694

\section{HAL Id: halshs-00865694 https://shs.hal.science/halshs-00865694}

Submitted on 24 Sep 2013

HAL is a multi-disciplinary open access archive for the deposit and dissemination of scientific research documents, whether they are published or not. The documents may come from teaching and research institutions in France or abroad, or from public or private research centers.
L'archive ouverte pluridisciplinaire HAL, est destinée au dépôt et à la diffusion de documents scientifiques de niveau recherche, publiés ou non, émanant des établissements d'enseignement et de recherche français ou étrangers, des laboratoires publics ou privés. 
Gilles Jacoud (ed.), Money and Banking in Jean-Baptiste Say's Economic Thought, London and New York: Routledge, 2013, xv + 266 pp., £ 85, ISBN: 978-0-415-67737-0

L'objectif de ce livre est de mettre à la disposition des lecteurs anglophones un choix des principaux textes de Jean-Baptiste Say qui traitent de la monnaie et des banques. La stratégie est claire : on dispose déjà de la traduction en anglais de certains des ouvrages de Say, par exemple de la première édition du Catéchisme de l'économie et de la quatrième édition du Traité. Ces textes ne seront pas repris car il s'agit, ici, de fournir aux lecteurs des documents auxquels ils ne peuvent pas avoir accès. Plutôt que de les regrouper par thème, l'éditeur, Gilles Jacoud, a décidé, à juste titre, de les regrouper par source. On retrouve dans ce recueil des extraits de la seconde édition du Catéchisme, de la première édition du Cours complet d'économie politique pratique, des éditions non traduites du Traité, de manuscrits et de textes divers.

Say pensait qu'il n'avait pas su donner dans la première édition du Catéchisme (1816) une forme appropriée à ses idées. II était donc opportun de traduire les chapitres XII et XIII de la seconde édition (1821) de cet ouvrage. Ces chapitres traitent respectivement de la monnaie et des signes représentatifs de la monnaie. Say y introduit une distinction qui est centrale dans son analyse. La monnaie est une marchandise qui a une valeur échangeable. Cette valeur, elle la tire, comme les autres marchandises, de ses usages: dans bien des cas, elle est indispensable quand on veut échanger les marchandises que l'on possède contre celles que l'on désire. Par opposition, les signes représentatifs de la monnaie - les billets au porteur, les effets de commerce - n'ont d'autre valeur que celle que leur procure la somme qu'ils donnent au porteur le droit de se faire payer.

Le Cours complet d'économie politique pratique (1828-9) n'a jamais été traduit en anglais. Say le présente comme un ouvrage destiné à un large public; mais il ne s'agit pas simplement comme dans le Catéchisme de vulgariser les enseignements de l'économie politique, il s'agit d'expliquer et c'est sans doute dans ce livre que Say expose le plus clairement les conclusions auxquelles, après bien des hésitations, il est parvenu à la fin de sa vie. Il ne convenait pas, ici, de se borner à des extraits et Jacoud propose une traduction de l'ensemble des chapitres du Cours qui traitent de la monnaie et des banques.

Le Traité d'économie politique pose un problème complexe : il fit l'objet de six éditions et les remaniements furent importants, ils traduisent l'évolution progressive des analyses de Say. La quatrième édition (1819) fut traduite en 1821 par C. Prinsep. Des rééditions de ce texte parurent aux États-Unis en 1821, 1824, 1827, 1830, 1832, 1834 et 1836. Elles donnèrent lieu à plusieurs réimpressions. Cependant ces ouvrages reprennent tous la traduction que Prinsep avait faite de la quatrième édition du Traité. C. Biddle qui les réalisa la compléta simplement en traduisant le discours préliminaire de Say, en supprimant les notes rédigées par Prinsep qu'il trouvait trop ricardiennes et en leur substituant ses propres remarques. Compte-tenu du fait que les lecteurs anglophones peuvent se reporter à ce texte, Jacoud a choisi de traduire, d'une part, des extraits des trois premières éditions et, d'autre part, des extraits des deux dernières. Le lecteur peut ainsi comprendre l'évolution de la pensée de Say ; mais il devra être attentif et, même, méticuleux.

Dans la dernière partie du recueil, on trouve une série de documents réunis sous le titre Manuscripts, notes and various texts. II s'agit, notamment, des notes que rédigea Say pour la 
seconde édition (1823) de la traduction française du Cours d'économie politique de Storch. Ces notes sont particulièrement intéressantes car, en critiquant certaines des idées défendues par Storch, Say est conduit à préciser sa pensée. On trouve aussi des textes inédits, notamment le plan pour un nouveau système monétaire où il reprend les propositions que Ricardo avait faites.

Jacoud présente ces textes d'excellente manière. Son point de départ est l'idée que c'est en étudiant les problèmes que connaissaient, alors, la France et l'Angleterre que Say élabora sa théorie monétaire. Progressivement, il construisit sa version de la théorie quantitative de la monnaie qui repose sur l'idée que la valeur de la monnaie est, comme celle des autres marchandises, déterminée par son offre et sa demande. Il distingue soigneusement les situations où les moyens de paiement sont convertibles en or ou en argent de celles où ils sont inconvertibles. Il admet qu'une variation de la quantité de monnaie affecte non seulement les prix mais la répartition des richesses et le niveau d'activité. Ceci justifie l'intervention de l'État et pose la question de savoir comment doit être organisé le système monétaire et bancaire.

La monnaie est une marchandise qui convient à tout le monde et qui peut, donc, servir d'intermédiaire dans les échanges. On la recherche non pour la consommer mais pour l'échanger contre un objet utile. Peu importe donc la matière dont elle est faite : elle peut être indifféremment d'or, d'argent ou de papier; elle remplira exactement le même office. Divers biens peuvent, simultanément, servir de moyens de paiement. On peut payer avec des pièces d'or, des billets, des lettres de change, des effets de commerce... Toutefois ces moyens de paiement diffèrent par leur nature. La monnaie proprement dite tire la valeur de ses usages. Ce n'est pas un signe, c'est une marchandise. II convient donc de distinguer le papier-monnaie inconvertible, qui tire sa valeur de l'usage que l'on en fait, du billet de banque qui est le signe de l'argent que l'on peut recevoir, quand on le veut, sur la présentation de cet effet. De la même façon, les lettres de change et les effets de commerce font office de monnaie et peuvent lui être substitués. Ils tirent leur valeur du fait qu'ils peuvent, quand ils arrivent à échéance, être échangés contre de la monnaie.

Quand on adopte comme monnaie des biens qui ont un autre usage, on en augmente considérablement la demande et la valeur. Si, par convention ou par coutume, un bien qui n'a pas d'autre emploi, est admis à faire office de monnaie, cette seule utilité suffit pour lui donner de la valeur. La valeur de la monnaie est déterminée par son offre et sa demande. Si, dans un pays, la production et la consommation deviennent plus considérables, la demande de monnaie augmente et, si la quantité en est restée la même, la valeur de chaque unité monétaire augmente. Réciproquement, si la quantité de monnaie s'accroît alors que sa demande reste inchangée, sa valeur diminue. Bien sûr, les effets seront différents selon qu'il s'agit d'une émission de papier-monnaie ou de billets de banque. Dans le premier cas, la monnaie se déprécie. Dans le second cas, cette dépréciation n'est que transitoire. Les pièces d'or ou d'argent qui valent alors moins que le métal dont elles sont faites, seront fondues et exportées jusqu'à ce que la quantité de moyens de paiement retrouve son niveau d'équilibre.

La monnaie n'est pas neutre. Une variation de sa valeur affecte la répartition des richesses. A la fin de sa vie, Say, s'appuyant sur Tooke, admet que l'émission de billets, réalisée le plus souvent lors des opérations d'escompte ou de prêt, diminue le taux d'intérêt. Pour ces deux raisons, une variation de la quantité de monnaie affecte le niveau d'activité. II explique qu'il peut exister, dans 
une dégradation légère et lente de la valeur de la monnaie, un avantage. Inversement, il soutient que, lorsqu'en 1816-1818 la Banque d'Angleterre réduisit la quantité de billets en circulation, l'industrie anglaise eut beaucoup à souffrir.

Il est ainsi conduit à développer une théorie monétaire des crises sur laquelle il s'appuie pour rendre compte de la crise commerciale que l'Angleterre connut en 1825 . Les banques - notamment les banques provinciales - ont utilisé la faculté qui leur a été accordée d'émettre des billets pour escompter une trop grande quantité d'effets de commerce. Les entrepreneurs ont ainsi pu donner à leurs affaires une proportion sans commune mesure avec leurs capitaux. La multiplication des billets a fait tomber la valeur de l'unité monétaire en-deçà de la valeur de l'or qui doit légalement s'y trouver. Les porteurs de billets ont donc demandé à être payés en or. La Banque d'Angleterre, contrainte d'acheter de l'or pour faire face à ses obligations, cessa d'émettre des billets et restreignit ses escomptes. Les négociants, privés des avances sur lesquelles ils comptaient, durent, quand les effets qu'ils avaient émis arrivèrent à échéance, vendre à perte les marchandises qu'ils avaient en stock. Ce fut le point de départ de la crise qui fut caractérisée par la faillite de nombreux banquiers qui, ayant mis en circulation des billets au porteur pour un montant excédant leurs capitaux propres, n'eurent pour faire face à leurs échéances que les engagements de leurs clients dont beaucoup avaient fait défaut.

Les conséquences d'une émission excessive de billets justifient, aux yeux de Say, l'idée que l'État doit intervenir pour imposer les règles que dicte la prudence. Mais que doit-il faire ? II faut arbitrer, d'une part, entre le coût de la monnaie et sa sûreté et, de l'autre, entre les risques de la concurrence et les inconvénients du monopole. Presque partout, les métaux précieux font office de monnaie et l'on peut penser que nul changement n'est, à cet égard, souhaitable. Cependant, un papier-monnaie peut très bien, à moindre coût, servir de moyen de paiement. Mieux, Say observe que son introduction en France ou en Angleterre fut, au moins dans un premier temps, favorable au développement de l'industrie. Ce qu'il craint c'est que les autorités ne résistent pas à la tentation de multiplier une marchandise qui ne coûte rien, ou presque, à produire et qu'elles ne lui fassent perdre ainsi toute sa valeur. La solution intermédiaire que proposait Ricardo le séduisit au point qu'il rédigea un plan qui en reprenait les propositions. Mais, dans les dernières éditions du Traité, il écarte cette idée qui ne lui semble réalisable que sous un gouvernement qui offrirait toutes les garanties désirables et au moyen d'une banque indépendante, c'est-à-dire dans des conditions politiques qui ne sont nullement satisfaites en France.

Say porte sur la Banque de France un regard plutôt critique : il lui reproche d'être trop prudente et de conserver dans ses coffres des sommes bien plus considérables que celles que la prudence lui conseille de tenir en réserve. Pire, elle ne prête qu'aux riches alors qu'il conviendrait qu'elle vienne en aide à des entrepreneurs dans l'embarras qui, sans être d'une sûreté parfaite, présentent, néanmoins, des garanties raisonnables. Mais l'exemple de l'Angleterre conduit Say à penser que l'on ne peut pas, sans risques, laisser se former un nombre indéfini de banques qui auraient toutes la possibilité d'émettre des billets au porteur. II préconise donc une solution intermédiaire : la Banque de France perdrait son privilège exclusif et serait exposée à la concurrence d'une ou deux banques d'émission. Il espère que ces établissements se feraient concurrence et que celui qui se rendrait le plus utile par les avances qu'il ferait au public serait dédommagé par une 
circulation plus étendue de ses billets au porteur. II voit la solution dans une liberté d'émission contrôlée.

Les contributions de Say au développement de la théorie de la monnaie et des banques méritent d'être étudiées et critiquées. L'intérêt du recueil qu'édite Jacoud est de les mettre à la disposition d'un plus large public.

Alain Béraud

THEMA, Université de Cergy-Pontoise, France 